Supporting Information

\title{
Source Apportionment of Sediment PAHs in Lake Calumet, Chicago: Application of Factor Analysis with Nonnegative Constraints
}

\author{
Philip A. Bzdusek and Erik R. Christensen* \\ Department of Civil Engineering and Mechanics and Center for Great Lakes Studies, \\ University of Wisconsin-Milwaukee, Milwaukee, WI 53201, USA \\ An Li and Qimeng Zou \\ School of Public Health, University of Illinois at Chicago, \\ 2121 West Taylor Street, MC-922, Chicago, Illinois 60612-7260
}

\section{Source Compositions}

For the purpose of this study, 12 published wood burning PAH references (32 PAH source profiles) were collected. Table S1 contains the wood type and measured phase (total or particulate) for each literature source profile. Collected literature data were corrected for particulate percentages using the $\mathrm{P} \%$ values, and normalized to the concentrations of $\mathrm{BeP}$. If $\mathrm{BeP}$ was absent, its concentration was assumed to be that of BaP. Results are summarized in Table S2. The results shown for wood burning I in Table S2 consider particulate phase source profiles only. Also, the oak wood profile from literature source S8 was eliminated because of an outlier value, chrysene (Chy).

\section{References}

S1. Khalili, N. R.; Scheff, P. A.; Holsen, T. M. Atmos. Environ. 1995, 29, 533-542.

S2. Jenkins, B. M.; Jones, A. D.; Turn, S. Q.; Williams, R. B. Atmos. Environ. 1996, 30, 3825-3835.

S3. Jenkins, B. M.; Jones, A. D.; Turn, S. Q.; Williams, R. B. Environ. Sci. Technol. 1996, 30, 2462-2469.

S4. $\quad$ Oanh, N. T. K.; Reutergårdh, L. B.; Dung, N. T. Environ. Sci. Technol. 1999, 33, 27032709.

S5. Chang, P. E. Masters Thesis, Illinois Institute of Technology, Chicago, IL, 1991.

S6. Hedberg, E.; Kristensson, A.; Ohlsson, M.; Johansson, C.; Johansson, P-A.; Swietlicki, E.; Vesely, V.; Wideqvist, U.; Westerholm, R. Atmos. Environ. 2002, 36, 4823-4837.

S7. McDonald, J. D.; Zielinska, B.; Fujita, E. M.; Sagebiel, J. C.; Chow, J. C.; Watson, J. G. Environ. Sci. Technol. 2000, 34, 2080-2091.

S8. Rogge, W. F.; Hildemann, L. M.; Mazurek, M. A.; Cass, G. R. Environ. Sci. Technol. 1998, 32, 13-22.

S9. Simoneit, B. R. T.; Rogge, W. F.; Lang, Q; Jaffé, R. Chemosphere: Global Change Science 2000, 2, 107-122.

S10. Padban, N.; Odenbrand, I. Energy Fuels 1999, 13, 1067-1073.

S11. Freeman, D. J.; Cattell, F. C. R. Environ. Sci. Technol. 1990, 24, 1581-1585.

S12. Fine, P. M.; Cass, G. R.; Simoneit, B. R. T. Environ. Sci. Technol. 2001, 35, 2665-2675. 
Table S1. Wood type and phase measured for each literature source.

\begin{tabular}{|c|c|c|}
\hline $\begin{array}{l}\text { Literature } \\
\text { Source }\end{array}$ & Wood Type & Phase \\
\hline $\mathrm{S} 1$ & oak & total \\
\hline $\mathrm{S} 2$ & almond prunings & particle \\
\hline $\mathrm{S} 2$ & douglas fir slash & particle \\
\hline $\mathrm{S} 2$ & ponderosa pine slash & particle \\
\hline $\mathrm{S} 3$ & almond & total \\
\hline S3 & walnut & total \\
\hline $\mathrm{S} 3$ & fir & total \\
\hline $\mathrm{S} 3$ & pine & total \\
\hline $\mathrm{S} 4$ & fuel & total \\
\hline S5 & wood & total \\
\hline S6 & average & total \\
\hline S6 & mixed hardwood & total \\
\hline S6 & oak & total \\
\hline S6 & pine & total \\
\hline S6 & eucalyptus & total \\
\hline S7 & softwood & total \\
\hline S7 & hardwood & total \\
\hline S7 & hardwood2 & total \\
\hline S7 & synthetic log & total \\
\hline S8 & pine wood & particle \\
\hline S8 & oak wood & particle \\
\hline S8 & synthetic log & particle \\
\hline S9 & pine & particle \\
\hline $\mathrm{S} 10$ & biomass & total \\
\hline S11 & wood & particle \\
\hline $\mathrm{S} 11$ & wood & particle \\
\hline $\mathrm{S} 12$ & red maple & particle \\
\hline $\mathrm{S} 12$ & n. red oak & particle \\
\hline $\mathrm{S} 12$ & paper birch & particle \\
\hline $\mathrm{S} 12$ & e. white pine & particle \\
\hline $\mathrm{S} 12$ & e. hemlock & particle \\
\hline $\mathrm{S} 12$ & balsam fir & particle \\
\hline
\end{tabular}


Table S2. Summary of Literature PAH source compositions normalized to Benzo[e]pyrene, Average \pm Standard Deviation (Number of Literature References, Number of Source Profiles)

\begin{tabular}{|c|c|c|c|}
\hline PAHs & $\mathrm{P} \%^{\mathrm{a}}$ & Wood Burning I $^{\mathrm{c}}$ & Wood Burning II \\
\hline $\mathrm{N}, \mathrm{M}^{\mathrm{b}}$ & & 5,14 & 12,32 \\
\hline Nap & $0.6 \%$ & $8.90 \pm 1.01(1,3)$ & $3.16 \pm 0.67(7,15)$ \\
\hline AcNP & $1.6 \%$ & $1.64 \pm 1.29(2,4)$ & $0.96 \pm 1.24(8,16)$ \\
\hline $\mathrm{AcN}$ & $2.3 \%$ & $0.24 \pm 0.00(1,1)$ & $0.66 \pm 0.09(7,13)$ \\
\hline $\mathrm{Fl}$ & $1.3 \%$ & $0.14 \pm 0.15(1,3)$ & $0.19 \pm 0.09(8,20)$ \\
\hline $\mathrm{PhA}$ & $3.4 \%$ & $5.35 \pm 6.89(4,12)$ & $3.33 \pm 0.98(11,30)$ \\
\hline An & $4.1 \%$ & $1.14 \pm 1.42(3,11)$ & $0.73 \pm 0.26(11,30)$ \\
\hline $\mathrm{FlA}$ & $10.3 \%$ & $14.92 \pm 16.52(5,14)$ & $7.86 \pm 4.56(12,32)$ \\
\hline Py & $14.3 \%$ & $13.54 \pm 12.24(5,14)$ & $7.43 \pm 4.46(12,32)$ \\
\hline $\mathrm{BaA}$ & $44.7 \%$ & $4.15 \pm 3.55(5,14)$ & $2.69 \pm 1.01(12,32)$ \\
\hline chy & $61.2 \%$ & $4.54 \pm 3.08(5,14)$ & $3.83 \pm 4.56(12,32)$ \\
\hline $\mathrm{BbFlA}$ & $87.8 \%$ & $1.64 \pm 1.08(5,14)$ & $1.93 \pm 1.31(11,29)$ \\
\hline $\mathrm{BkFlA}$ & $88.9 \%$ & $1.79 \pm 0.83(4,13)$ & $1.59 \pm 0.60(11,28)$ \\
\hline $\mathrm{Bb}+\mathrm{kFlA}$ & $89.0 \%$ & $3.31 \pm 1.77(5,14)$ & $3.16 \pm 1.20(12,30)$ \\
\hline $\mathrm{BeP}$ & $91.1 \%$ & $1.00 \pm 0.00(4,12)$ & $1.00 \pm 0.00(10,29)$ \\
\hline $\mathrm{BaP}$ & $93.3 \%$ & $1.55 \pm 0.50(5,14)$ & $1.41 \pm 0.40(12,32)$ \\
\hline IP & $96.7 \%$ & $1.13 \pm 0.62(4,11)$ & $1.07 \pm 0.53(8,22)$ \\
\hline $\mathrm{dBahA}$ & $97.4 \%$ & $0.13 \pm 0.10(3,9)$ & $0.26 \pm 0.11(5,12)$ \\
\hline BghiP & $93.8 \%$ & $0.73 \pm 0.30(5,13)$ & $0.68 \pm 0.25(9,22)$ \\
\hline
\end{tabular}

a. $\quad \mathrm{P} \%=$ Percent in particulate phase in air. See Supporting Information for Li et. al [15] for literature sources.

b. Number of literature references, number of wood profiles considered.

c. Particulate phase only in original references, references S2, S8, S9, S11, and S12. 